\title{
Benzodiazepine Use and Risk of Dementia in the Elderly Population: A Systematic Review and Meta-Analysis
}

\author{
Md. Mohaimenul Islam ${ }^{a, b} \quad$ Usman lqbal $^{c}$ Bruno Walther ${ }^{c}$ Suleman Atique ${ }^{a, b}$ \\ Navneet Kumar Dubey ${ }^{d}$ Phung-Anh Nguyen ${ }^{a, b}$ Tahmina Nasrin Poly ${ }^{f}$ \\ Jakir Hossain Bhuiyan Masud ${ }^{a, b}$ Yu-Chuan (Jack) Li ${ }^{a, b, e}$ Syed-Abdul Shabbir ${ }^{a, b}$ \\ ${ }^{a}$ Graduate Institute of Biomedical Informatics, College of Medical Science and Technology, ${ }^{b}$ International Center \\ for Health Information Technology (ICHIT), ' Aster Program in Global Health and Development, College of Public \\ Health, and ${ }^{d}$ Graduate Institute of Biomedical Materials and Tissue Engineering, College of Biomedical Engineering, \\ Taipei Medical University, and ${ }^{\mathrm{e} C h a i r, ~ D e p a r t m e n t ~ o f ~ D e r m a t o l o g y, ~ W a n ~ F a n g ~ H o s p i t a l, ~ T a i p e i, ~ a n d ~}{ }^{\mathrm{f}}$ Department of \\ Microbiology and Immunology, Tzu Chi University, Hualien, Taiwan
}

\section{Key Words}

Benzodiazepines · Dementia - Diabetic · Hypertension .

Heterogeneity

\begin{abstract}
Background: Benzodiazepines are a widely used medication in developed countries, particularly among elderly patients. However, benzodiazepines are known to affect memory and cognition and might thus enhance the risk of dementia. The objective of this review is to synthesize evidence from observational studies that evaluated the association between benzodiazepines use and dementia risk. Summary: We performed a systematic review and metaanalysis of controlled observational studies to evaluate the risk of benzodiazepines use on dementia outcome. All control observational studies that compared dementia outcome in patients with benzodiazepine use with a control group were included. We calculated pooled ORs using a random-effects model. Ten studies (of 3,696 studies identified) were included in the systematic review, of which 8 studies were included in random-effects meta-analysis
\end{abstract}

and sensitivity analyses. Odds of dementia were $78 \%$ higher in those who used benzodiazepines compared with those who did not use benzodiazepines (OR 1.78; $95 \% \mathrm{Cl}$ 1.33-2.38). In subgroup analysis, the higher association was still found in the studies from Asia (OR 2.40; $95 \% \mathrm{Cl} 1.66-$ 3.47) whereas a moderate association was observed in the studies from North America and Europe (OR 1.49; $95 \% \mathrm{Cl}$ 1.34-1.65 and OR 1.43; 95\% Cl 1.16-1.75). Also, diabetics, hypertension, cardiac disease, and statin drugs were associated with increased risk of dementia but negative association was observed in the case of body mass index. There was significant statistical and clinical heterogeneity among studies for the main analysis and most of the sensitivity analyses. There was significant statistical and clinical heterogeneity among the studies for the main analysis and most of the sensitivity analyses. Key Messages: Our results suggest that benzodiazepine use is significantly associated with dementia risk. However, observational studies cannot clarify whether the observed epidemiologic association is a causal effect or the result of some unmeasured confounding variable. Therefore, more research is needed.

(c) 2016 S. Karger AG, Basel

\section{KARGER}

(c) 2016 S. Karger AG, Basel

E-Mail karger@karger.com

www.karger.com/ned
Dr. Syed-Abdul Shabbir, MD, MSc, PhD

Assistant Professor and Health IT consultant, Graduate Institute of Biomedical Informatics College of Medical Science and Technology, Taipei Medical University $250 \mathrm{Wu}-\mathrm{Hsing}$ St., Taipei 110 (Taiwan)

E-Mail drshabbir@tmu.edu.tw 


\section{Introduction}

The prevalence of dementia is increasing dramatically worldwide, but the types of dementia vary between countries [1]. Alzheimer disease is the most common type of dementia in Western countries, whereas vascular dementia is more common in Asia [2]. Up to 35.6 million people were afflicted with dementia in 2012, and this number is expected to double by 2030 [3]. The prevalence of dementia varies considerably by age group; a higher prevalence in patients above the age of 85 years was found than in patients above the age of 65 years [4]. Alzheimer disease remains the most common cause of dementia, and several risk factors are associated with dementia, such as age, gender, education [5], hormonal effects, head trauma [6], depression [7], stroke [8], and blood pressure [9].

Benzodiazepines are a type of medication acting on the central nervous system and enhance the effect of the neurotransmitter gamma-aminobutyric acid. Benzodiazepines are a popular medication in the treatment of anxiety, depression, and insomnia patients around the world [10]. In 2007, nearly 85 million benzodiazepine prescriptions were provided in United States to outpatients with anxiety and depression [11] which was a little less than the 2001 account of 90 million [12]. In Germany and Canada, the percentage of benzodiazepine prescription is $18.9 \%$ [10] and $15.0 \%$ [13], respectively, of the total of described depression drugs; furthermore, women were prescribed more benzodiazepine compared to men, and prescription was higher in the older population as compared with the younger population [14]. However, among older users, the use of benzodiazepine causes serious adverse effects including impaired cognitive function.

To our knowledge, no systematic review of the association between the use of benzodiazepines and the risk of dementia among elderly patients has been published so far. Therefore, we conducted a systematic review and meta-analysis of observational studies to determine whether the long-term use of benzodiazepines is associated with an increased risk of dementia among older patients when compared to non-benzodiazepine users. In our review, we only included studies that mentioned patients having exposure to benzodiazepine for $\geq 30$ days and included patients over the age of 55 years. To identify patients of particularly high risk, we conducted an additional analysis to determine whether dementia risk is associated with gender, geographical area, smoking status, comorbidity, and co-medication.

\section{Methods}

This systematic review and meta-analysis was conducted in accordance with the Preferred Reporting Items for Systematic Reviews and Meta-Analyses (PRISMA) [15]. A review written protocol was not drafted.

\section{Eligibility Criteria}

We included only those studies that met the following criteria: (1) the study reported separately the relevant risk of benzodiazepine use and dementia diagnosis based on an observational study design, (2) one of the exposure outcomes of interest was benzodiazepine, (3) the outcome of interest was dementia, and (4) the study provided an OR or a risk ratio with a $95 \%$ CI or provided sufficient information to calculate them.

We excluded any study that met the following criteria: (1) the study was a case-report, editorial, review, or clinical trial; and (2) the outcome of interest was not dementia.

\section{Data Sources}

A comprehensive search of MEDLINE, EMBASE, Cochrane Central Register of Controlled Trials, and Database of Systematic Reviews from the earliest available online year of indexing up to June 2016 was conducted. Our search strategies included controlled vocabulary and related keywords for 2 concepts: "dementia" and "benzodiazepines use." We included all study designs, publication years, languages, and inclusion of human participants in our search. Furthermore, the authors of potentially eligible abstracts, posters, or manuscripts were contacted via email to possibly obtain published information, and thus additional data was obtained. No unpublished data were included in our analysis.

Data Collection and Analysis

Study Selection

Two of our authors (Md.M.I. and P.-A.N.) independently conducted the article selection, data extraction, and assessment of risk of bias, and they also followed guidance from the Cochrane Collaboration to include observational studies to maximize study, participant, and event inclusion into our analysis which allowed us to also conduct sensitivity and subgroup analyses. All disagreements between these 2 authors were resolved by consensus.

\section{Data Extraction}

Ten studies that fulfilled all of our criteria as stated above were then independently entered into our database by the same 2 authors, with the following entries: first author's last name, publication year, country source of study, participants' characteristics, method of ascertainment of dementia, sample size, variable adjustment, and the dementia risk estimated with $95 \%$ CI. We also screened the title, abstract, and full text in a similar fashion; however, specific exclusion reasons were documented only during full text screening. Upon selection of the final group of studies, the same 2 authors independently extracted the qualitative and quantitative data using a standardized data extraction form adjudicated by a third author (S.-A.S).

\section{Assessment of Methodological Quality}

The main outcome of this meta-analysis was the possible association of benzodiazepine use and dementia risk among the elderly population. We assessed the methodological quality of the included observational studies based on a modified version of the 
Newcastle-Ottawa Scale (NOS). In the case of a case-control study, NOS consists of 8 items with 3 subscales, and the total maximum score of these 3 subsets is 9 . We considered a study which scored $\geq 7$ a high-quality study since a standard criterion for what constitutes a high-quality study has not yet been universally established. The mean value for the 8 studies assessed by us was 8.25 .

\section{Statistical Analysis}

To test for the association between benzodiazepine use and the risk of dementia, we analyzed our data with the Review Manager program (version 5.3). We also reported measures of association extracted from the included studies using the ORs with a 95\% CI. For studies that did not report a measure of association between benzodiazepine exposure and dementia but did include the required information, we calculated an unadjusted OR using the Peto method. In the case of a systematic analysis, we examined study countries, study design, study period, number of cases, and control and adjustments. To resolve the proportion of variability in the effect estimates due to heterogeneity, we used the Cochrane's $\mathrm{Q}$ test (reported with a $\lambda^{2}$ and $p$ value) and the $I^{2}$ statistic [16]. We then used the accepted cutoff of $50 \%$ to define significant heterogeneity and additionally calculated the associated $p$ value (via a $\chi^{2}$ test).

To explore the heterogeneity between studies, we conducted an analysis stratified by gender, age, geographical location, body mass index (BMI), comorbidity, and co-medication. Furthermore, we evaluated the impact of adjustment for smoking and drinking status on the association between benzodiazepine use and the risk of dementia. We performed subgroup analysis by excluding each study individually to assess its influence on the overall result of our meta-analysis.

Finally, we generated forest plots for each of our exposure-outcome comparisons, and we used a random-effects-based model to calculate the weighted mean, OR, associated $95 \% \mathrm{CI}$, and $p$ value due to significant clinical, methodological, and statistical heterogeneity across the included studies. Thus, all effect estimates were assumed to approximate the OR and were pooled in the metaanalysis.

\section{Results}

\section{Study Selection}

We identified 1,318 unique titles and abstracts, of which 1,295 studies were excluded based on our predetermined eligibility criteria described above. The remaining 23 studies underwent full-text screening but only 10 studies ultimately met all of our criteria for inclusion in our systematic review; furthermore, 8 of out these 10 studies were included in our meta-analysis. The most common reason for exclusion of the 13 excluded studies was the impossibility to extract data $(n=5)$, followed by dementia not being measured $(n=3)$, and full-text duplication $(n=2)$. Figure 1 summarizes our selection process.

Benzodiazepine Use and Risk of Dementia

\section{Systematic Review}

A qualitative synthesis of the 10 studies meeting all of our inclusion criteria is summarized in Table 1. Six studies were case-control design studies [17-22] and 4 were cohort studies [23-26]. The included studies were published between 1983 and 2013, thus spanning 31 years. Five studies were conducted in Europe [18, 19, 24-26], 3 in Asia [17, 21, 22], and the remaining 2 in North America [20,23]. Eight studies reported that the age at the onset of dementia was over 65 years but 2 studies reported an age of onset below 60 years [24, 25].

Most of the studies mentioned benzodiazepines exposure but they did not evaluate risk according to individual drugs in the benzodiazepine group. However, one case-control study mentioned zolpidem [17]. Two studies included exposed participants who were treated with both benzodiazepine and psychotropic medicine. Eight studies used pharmacy and prescription records $(80 \%)$ to identify benzodiazepine exposure while 2 studies used diagnosis code (20\%) to identify dementia.

A total of 101,659 study subjects were included in our quantitative synthesis of which 23,773 and 48,773 were men and women, respectively; the discrepancy in numbers is due to 2 studies which did not mention gender information. The total number of dementia patients in the included studies was 35,482. All studies controlled for age and gender; each study also adjusted for multiple other potential confounding factors, including medications and comorbidities, but the precise extent of statistical adjustment varied by study as noted in Table 1 . Furthermore, 3 studies mentioned dose-response relationship, and 2 studies recorded long- and short-life benzodiazepine association.

\section{Meta-Analyses}

Our meta-analysis pooled data from 8 of the 10 studies included in the systematic review. These 8 studies included 66,177 participants and 30,914 cases of dementia; the remaining 2 studies were not included because of their study design and substantially overlapping study population.

Our primary meta-analysis of the 8 observational studies found that benzodiazepine use significantly increased dementia risk (Fig. 2). The pooled summary OR was 1.78 (95\% CI 1.33-2.38) in a random-effects model for benzodiazepine users as compared to benzodiazepine non-users. There was significant heterogeneity among the studies $\left(Q=534.13, p<0.00001, I^{2}=99 \%\right)$. For the association between benzodiazepine use and dementia risk, subgroup analysis was adopted according to stratification on gen- 
Fig. 1. Diagram of study selection, adapted

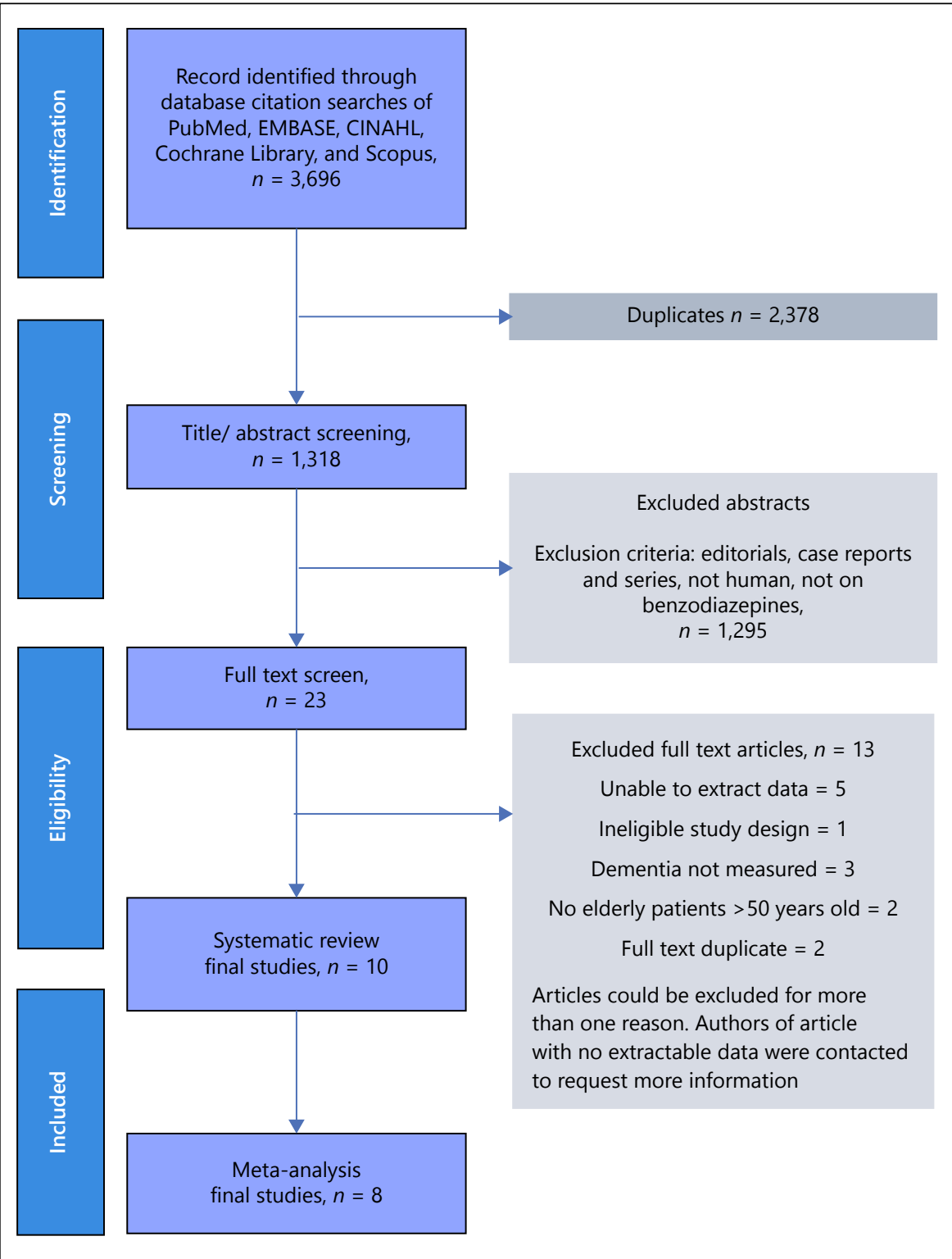
from PRISMA group 2009 flow diagram.

der, geographical location, BMI, smoking and drinking status, comorbidity, and co-medication (Table 2). We also found that women had a significantly higher risk of developing dementia than men; the pooled summary for female patients was OR 1.16 (95\% CI 1.00-1.36). The 6 case-control studies (OR 1.89; 95\% CI 1.34-2.68) and the 2 population-based studies (OR 1.47; 95\% CI 1.28-1.69) demonstrated the significant relationship between benzodiazepine use and dementia risk, although the numbers differed somewhat. In patients whose BMI was greater than 30 , benzodiazepine use was associated with a lower dementia risk (OR 0.96; 95\% CI 0.84-1.09); however, since the CI included no effect, this association was not significant. Concerning the geographical location, benzodiazepine use significantly increased dementia risk in European populations (OR 1.43; 95\% CI 1.16-1.75), and this association was even stronger in North American (OR 1.49; 95\% CI 1.34-1.65) and Asian (OR 2.40; 95\% CI 1.66-3.47) populations. Only 2 studies considered smoking status; while we found a negative relationship for past smokers (OR 0.89; 95\% CI 0.63-1.27), we found a positive relationship for non-smokers (OR 1.08; 95\% CI 0.71-1.65) and current smokers (OR 1.13; 95\% CI 0.80-1.60). However, none of these 3 relationships were significant. Likewise, 


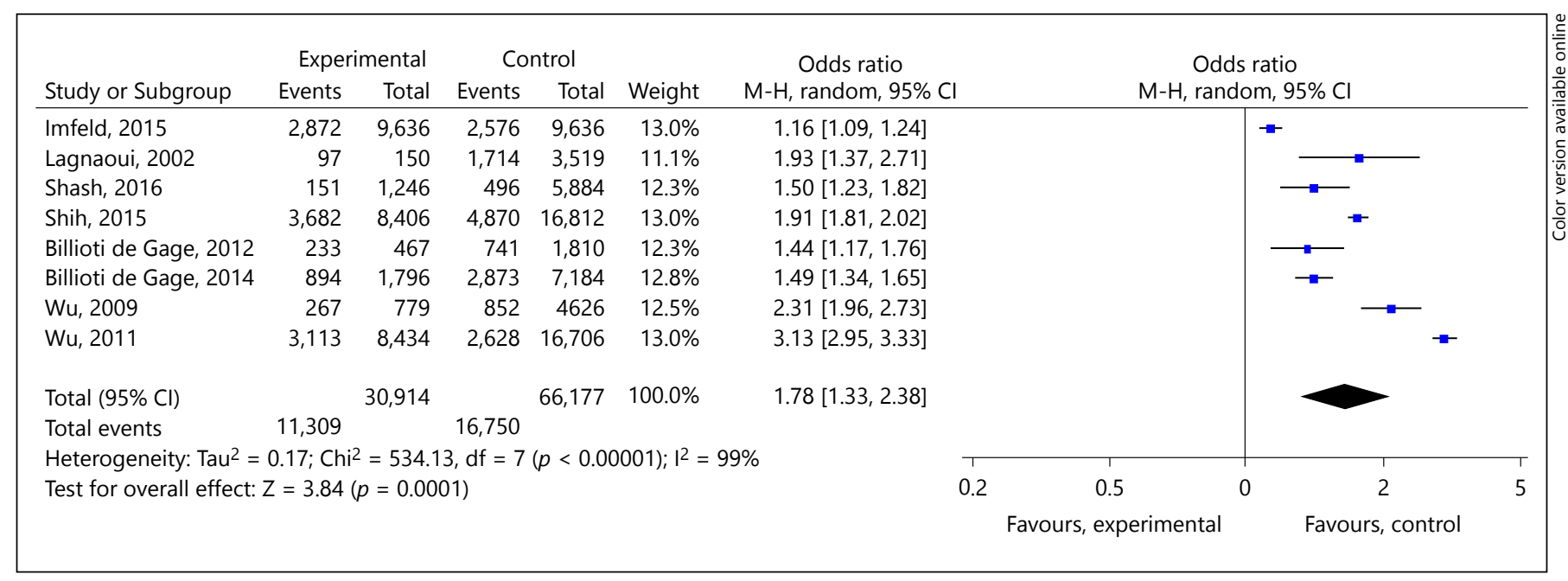

Fig. 2. ORs and 95\% CIs from studies of risk of dementia in patients receiving benzodiazepines.

Table 1. Characteristics of 10 studies included in our systematic analysis and meta-analysis

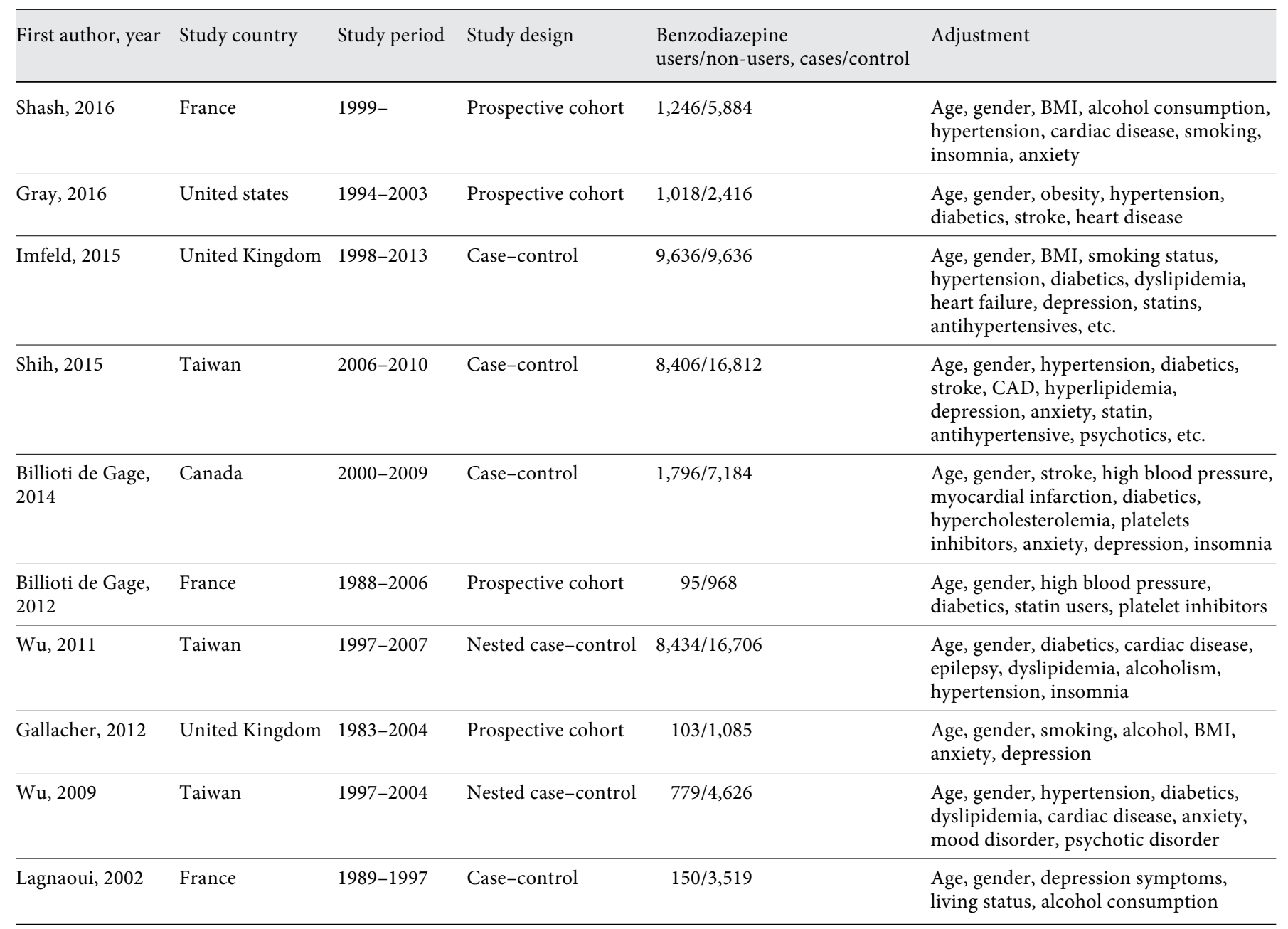


Table 2. Subgroup analysis of the association between benzodiazepines use and dementia risk

\begin{tabular}{|c|c|c|c|c|c|}
\hline Category variables & $\begin{array}{l}\text { Number } \\
\text { of studies }\end{array}$ & OR (95\% CI) & $I^{2}, \%$ & $\begin{array}{l}p \text { value of } \\
\text { heterogeneity }\end{array}$ & Method \\
\hline \multicolumn{6}{|l|}{ Gender } \\
\hline Male & 3 & $1.00(0.96-1.04)$ & 0 & 1.00 & Random \\
\hline Female & 6 & $1.16(1.00-1.36)$ & 95 & $<0.00001$ & Random \\
\hline \multicolumn{6}{|l|}{ Geographic location } \\
\hline Asia & 3 & $2.40(1.66-3.47)$ & 99 & $<0.00001$ & Random \\
\hline Europe & 4 & $1.43(1.16-1.75)$ & 100 & 0.001 & Random \\
\hline \multicolumn{6}{|l|}{ BMI } \\
\hline$>30$ & 2 & $0.96(0.84-1.09)$ & 49 & 0.16 & Random \\
\hline \multicolumn{6}{|l|}{ Smoking status } \\
\hline Non-smoker & 2 & $1.08(0.71-1.65)$ & 97 & $<0.000001$ & Random \\
\hline Current smoker & 2 & $1.13(0.80-1.60)$ & 83 & 0.02 & Random \\
\hline Past smoker & 2 & $0.89(0.63-1.27)$ & 96 & $<0.000001$ & Random \\
\hline \multicolumn{6}{|l|}{ Drinking status } \\
\hline Drinker & 4 & $1.06(0.61-1.86)$ & 97 & $<0.00001$ & Random \\
\hline Wine & 3 & $0.81(0.70-0.93)$ & 30 & 0.24 & Random \\
\hline \multicolumn{6}{|l|}{ Comorbidity } \\
\hline Diabetics & 5 & $1.30(0.98-1.72)$ & 96 & $<0.00001$ & Random \\
\hline Hypertension & 4 & $1.30(0.84-2.00)$ & 98 & $<0.00001$ & Random \\
\hline Cardiac disease & 3 & $1.13(0.82-1.55)$ & 88 & 0.0002 & Random \\
\hline Dyslipidemia & 2 & $1.29(1.18-1.41)$ & 69 & 0.07 & Random \\
\hline \multicolumn{6}{|l|}{ Co-medication } \\
\hline Statin & 2 & $1.41(1.32-1.50)$ & 0 & 0.99 & Random \\
\hline \multicolumn{6}{|l|}{ Others } \\
\hline Depression & 5 & $1.64(0.96-2.80)$ & 99 & $<0.00001$ & Random \\
\hline Anxiety & 3 & $1.75(1.55-1.98)$ & 74 & 0.02 & Random \\
\hline Insomnia & 3 & $1.91(1.38-2.63)$ & 94 & $<0.00001$ & Random \\
\hline Psychiatry & 2 & $4.14(0.55-30.84)$ & 96 & $<0.00001$ & Random \\
\hline
\end{tabular}

drinking alcohol was not significantly associated with dementia risk (OR 1.06; 95\% CI 0.61-1.86) but wine consumption significantly decreased dementia risk (OR 0.81; 95\% CI 0.70-0.93). We also investigated the impact of 4 comorbidities (namely, hypertension, diabetics, dyslipidemia, and cardiac disease). Hypertension and diabetics were positively associated with dementia risk (OR 1.30; 95\% CI 0.84-2.00 and OR 1.30; 95\% CI 0.98-1.72, respectively). Likewise, dyslipidemia was moderately associated with dementia risk (OR 1.29; 95\% CI 1.18-1.41), and cardiac disease was also slightly associated with dementia risk (OR 1.13; 95\% CI 0.82-1.55). In addition, we also found a significant association in the case of hypocholesterolemia, arterial fibrillation, epilepsy, Parkinson disease, and cerebrovascular condition.

As for the adjustment of other dementia risk factors, the use of statin drugs significantly increased dementia risk (OR 1.41; 95\% CI 1.32-1.50). Similarly, the use of anti-hypertensive medicine, platelets aggregation, anticoagulant, and platelets inhibitors were also considered as risk factors. Furthermore, psychiatric patients who took benzodiazepine were highly associated with dementia risk (OR 4.14; 95\% CI 0.55-30.84). Likewise, depression, anxiety, and insomnia patients were also significantly associated with dementia risk (Table 2). In the subgroup meta-analysis, we also found a significant positive association of benzodiazepine use and dementia risk. Taken together, our results suggest that the long-term use of benzodiazepines under most circumstances increases a patient's risk of developing dementia.

\section{Sensitivity Analysis}

To assess whether a single study had a substantial influence on the main results, we excluded each study and evaluated its effect on the summary estimates and heterogeneity of the main analysis. We performed sensitivity analysis to evaluate permanent use and current use of benzodiazepine use and dementia risk. For the association of short half-life ( $\leq 20 \mathrm{~h}$ ) and long half-life benzodiazepine drugs, we also found significant risk of developing dementia. 


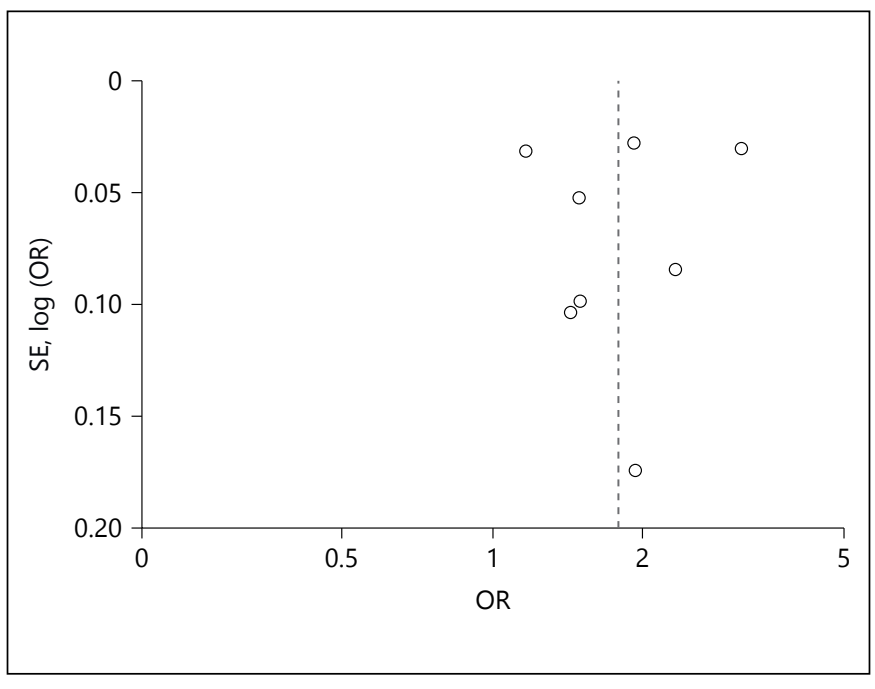

Fig. 3. Finnel plot shows association between benzodiazepines use and patients dementia.

\section{Publication Bias}

When the number of studies is small, the visual interpretation and test for asymmetry of the funnel plot of the publications are not very reliable [27]. Figure 3 shows the funnel plot which indicates the existence of some publication bias. The Egger's regression test of the funnel asymmetry showed a highly significant publication bias ( $p$ value $<0.05)$.

\section{Discussion}

\section{Main Findings}

This systematic review of 10 studies and meta-analysis of 8 studies demonstrated a 1.78 -fold increased risk of developing dementia in patients who used benzodiazepines for $>30$ days, and usually considerably longer. However, we interpret these results with caution for 2 reasons: (1) we found significant statistical and clinical heterogeneity among the studies, and (2) the inability of this type of study to distinguish between causal relationships or mere statistical association, with the possibility of confounding variables causing the observed relationship. Nevertheless, our results indicate an increased risk of the development of dementia in patients who use benzodiazepines, no matter what the ultimate causal relationship is. Furthermore, this relationship holds for benzodiazepine use alone, or in association with other diseases, such as diabetics, dyslipidemia, arterial fibrillation, hypertension, stroke, cardiac disease, hyperlipidemia, hypocholes- terolemia, epilepsy, insomnia, and anxiety (Table 3). This relationship is further associated with other factors, such as age, gender, smoking and drinking status, long- and short-acting benzodiazepine drugs, statin drugs, platelets aggregation, anti-coagulant and anti-hypertensive drugs. Our result is further supported by one previous metaanalysis from China which also found that long-term benzodiazepine users have an increased risk of developing dementia when compared with non-users [28].

The etiology of dementia is heterogeneous. There is no clear potential pathological hypothesis that could explain how benzodiazepines could facilitate or trigger the onset of dementia, by either some lasting neurochemical or neurotoxic effect, or by their negative cognitive effects. However, some studies suggested that benzodiazepines could precipitate the onset of dementia or mental decline by altering or impairing higher mental activities. Along these lines, Saczynski et al. [7] mentioned that depressive symptoms were associated with an increased risk of subsequent cognitive decline, and Lara et al. [29] found that high levels of cortisol may be associated with depression and stress and may thus cause neural death. Furthermore, a study reported an increased risk of stroke and coronary heart disease in patients with the ApoE polymorphism, a known genetic risk factor for dementia [30].

In the subgroup analysis, we found diabetes and hypertension were significantly associated with dementia risk. Yamada et al. [31] pointed out that hypertension is a primary risk factor for dementia but not a risk factor for Alzheimer disease [32]. Type 2 diabetics are associated with insulin resistance; because of this, it is related to the formation of $A \beta$ and inflammatory agents in the brain [33], which leads to an increased dementia risk. Likewise, patients with a history of depression, psychotic disorder, and head injury are also at risk of developing dementia in later life [7].

Included studies suggested that sleep disturbance was common in the prodromal phase of dementia, and therefore, benzodiazepines were prescribed for such early symptoms of dementia. Studies also mentioned that the length of time between the initial prescription and index diagnosis (i.e., the onset of dementia) was relatively long (meaning, 4 years). However, it might be difficult to determine whether, and to what extent, the prescription of benzodiazepines might be a consequence of prodromal symptoms of dementia. Johansson et al. [34] mentioned that persistent mid-life anxiety and depression could be associated with a higher chance of dementia in older people. Future research should therefore consider a long follow-up of at least 15-20 years because then it would be possible to evaluate the risk of long-term use of benzodi- 
Table 3. Univariate analysis: association between benzodiazepine use and dementia risk

\begin{tabular}{|c|c|c|c|c|}
\hline Study & Variables & Case/use & $\begin{array}{l}\text { Control/ } \\
\text { non-use }\end{array}$ & OR \\
\hline \multirow[t]{17}{*}{ Shash, 2016} & $\mathrm{BMI}>30$ & 165 & 746 & $1.05(0.88-1.26)$ \\
\hline & Alcohol consumption & & & \\
\hline & Drinker & 966 & 4,816 & $0.77(0.66-0.89)$ \\
\hline & Ex-drinker & 235 & 923 & $1.37(1.17-1.60)$ \\
\hline & Non drinker & 42 & 132 & $1.52(1.07-2.16)$ \\
\hline & Smoking status & & & \\
\hline & Non & 820 & 3,462 & $1.35(1.18-1.53)$ \\
\hline & Current & 65 & 331 & $0.92(0.70-1.21)$ \\
\hline & Past & 361 & 2,089 & $0.74(0.65-0.85)$ \\
\hline & Comorbidity & & & \\
\hline & Hypertension & 835 & 3,481 & $1.40(1.23-1.60)$ \\
\hline & Depression & 247 & 532 & $2.49(2.11-2.93)$ \\
\hline & Diabetics & 96 & 452 & $1.00(0.80-1.26)$ \\
\hline & Hypocholesterolemia & 502 & 2,084 & $1.23(1.09-1.39)$ \\
\hline & Cardiac disease & 149 & 494 & $1.48(1.22-1.80)$ \\
\hline & Insomnia & 374 & 798 & $2.73(2.37-3.15)$ \\
\hline & Anxiety & 416 & 1,185 & $1.99(1.74-2.27)$ \\
\hline \multirow[t]{19}{*}{ Imfeld, 2015} & $\mathrm{BMI}>30$ & 1,337 & 1,446 & $0.91(0.84-0.99)$ \\
\hline & Short acting & 895 & 877 & $1.02(0.93-1.13)$ \\
\hline & Long acting & 722 & 706 & $1.02(0.92-1.14)$ \\
\hline & Smoking status & & & \\
\hline & Non-smoker & 4,500 & 4,827 & $0.87(0.82-0.92)$ \\
\hline & Current smoker & 1,063 & 828 & $1.32(1.20-1.45)$ \\
\hline & Ex-smoker & 3,390 & 3,261 & $1.06(1.00-1.13)$ \\
\hline & Comorbidity & & & \\
\hline & Arterial & 5,376 & 4,971 & $1.18(1.12-1.25)$ \\
\hline & Diabetics & 1,482 & 1,085 & $1.43(1.32-1.56)$ \\
\hline & Dyslipidemia & 1,651 & 1,390 & $1.23(1.13-1.33)$ \\
\hline & Atrial fibrillation & 1,316 & 886 & $1.56(1.43-1.71)$ \\
\hline & Heart failure & 757 & 616 & $1.25(1.12-1.39)$ \\
\hline & Depression & 1,198 & 1,190 & $1.01(0.92-1.10)$ \\
\hline & Co-medication & & & \\
\hline & Anti-hypertensive & 6,607 & 6,185 & $1.22(1.15-1.29)$ \\
\hline & Statins & 3,243 & 2,551 & $1.41(1.32-1.50)$ \\
\hline & Platelets aggregation & 4,476 & 3,420 & $1.58(1.49-1.67)$ \\
\hline & Anticoagulant & 819 & 536 & $1.58(1.41-1.77)$ \\
\hline \multirow[t]{11}{*}{ Billioti de Gage, 2014} & Short half-life $(<20 \mathrm{~h})$ & 585 & 1,996 & $1.26(1.12-1.40)$ \\
\hline & Long half-life $(>20 \mathrm{~h})$ & 309 & 877 & $1.49(1.30-1.72)$ \\
\hline & Comorbidity & & & \\
\hline & High blood pressure & 1,155 & 4,508 & $1.07(0.96-1.19)$ \\
\hline & Myocardial infraction & 61 & 330 & $0.73(0.55-0.96)$ \\
\hline & Stroke & 125 & 416 & $1.22(0.99-1.50)$ \\
\hline & Hypercholesterolemia & 376 & 1,187 & $1.34(1.18-1.52)$ \\
\hline & Diabetics & 336 & 1,299 & $1.04(0.91-1.19)$ \\
\hline & Anxiety & 384 & 1,083 & $1.53(1.35-1.74)$ \\
\hline & Depression & 52 & 172 & $1.22(0.89-1.66)$ \\
\hline & Insomnia & 72 & 229 & $1.27(0.97-1.66)$ \\
\hline \multirow[t]{8}{*}{ Billioti de Gage, 2012} & Wine consumption & 267 & 1,066 & $0.93(0.76-1.14)$ \\
\hline & Comorbidity & & & \\
\hline & Depression & 46 & 153 & $1.18(0.84-1.67)$ \\
\hline & High blood pressure & 274 & 1,125 & $0.86(0.70-1.06)$ \\
\hline & Diabetics & 29 & 96 & $1.18(0.77-1.81)$ \\
\hline & Co-medication & & & \\
\hline & Statins & 26 & 73 & $1.40(0.89-2.22)$ \\
\hline & Platelets inhibitors & 32 & 164 & $0.74(0.50-1.09)$ \\
\hline
\end{tabular}


Table 3. (continued)

\begin{tabular}{|c|c|c|c|c|}
\hline Study & Variables & Case/use & $\begin{array}{l}\text { Control/ } \\
\text { non-use }\end{array}$ & OR \\
\hline \multirow[t]{2}{*}{ Wu, 2009} & $\begin{array}{l}\text { Cumulative dosage of BZD use } \\
90 \text { DDD < cumulative dose }< \\
180 \text { DDD }\end{array}$ & 85 & 389 & $1.28(0.97-1.68)$ \\
\hline & Cumulative dose $>180$ DDD & 267 & 885 & $1.39(1.12-1.73)$ \\
\hline \multirow[t]{13}{*}{$\mathrm{Wu}, 2011$} & Female & 4,347 & 8,591 & $1.00(0.95-1.06)$ \\
\hline & \multicolumn{4}{|l|}{ Comorbid condition } \\
\hline & Diabetics & 2,602 & 3,090 & $1.97(1.85-2.09)$ \\
\hline & Cerebrovascular disease & 3,736 & 3,419 & $3.09(2.92-3.27)$ \\
\hline & Hypertension & 4,953 & 6,653 & $2.15(2.04-2.27)$ \\
\hline & Dyslipidemia & 2,182 & 3,444 & $1.34(1.26-1.43)$ \\
\hline & Epilepsy & 294 & 118 & $5.04(4.09-6.30)$ \\
\hline & Parkinsonism & 990 & 471 & $4.58(4.09-5.13)$ \\
\hline & Alcoholism & 186 & 151 & $2.47(1.99-3.07)$ \\
\hline & Mood disorder & 1,217 & 831 & $3.22(2.94-3.53)$ \\
\hline & Anxiety disorder & 2,375 & 3,035 & $1.77(1.66-1.88)$ \\
\hline & Psychotic disorder & 269 & 49 & $11.20(8.25-15.20)$ \\
\hline & Sleep disorder & 3,874 & 5,187 & $1.89(1.79-2.00)$ \\
\hline \multirow[t]{7}{*}{ Lagnaoui, 2002} & Depression & 44 & 722 & $1.61(1.12-2.31)$ \\
\hline & Psychotic disorder & 9 & 148 & $1.45(0.73-2.91)$ \\
\hline & Wine consumption & 68 & 1,886 & $0.72(0.52-1.0)$ \\
\hline & \multicolumn{4}{|l|}{$\begin{array}{l}\text { Educational level (years of } \\
\text { schooling) }\end{array}$} \\
\hline & Low $(\leq 5$ years $)$ & 126 & 2,496 & $2.15(1.38-3.35)$ \\
\hline & Middle (6-11) & 19 & 780 & $0.51(0.31-0.83)$ \\
\hline & High $>12$ years & 5 & 243 & $0.46(0.19-1.14)$ \\
\hline
\end{tabular}

azepines in younger adults as well as to better assess the exact role of anxiety, sleep disorders, and depression as early risk factors of future dementia development.

\section{Limitations}

Our systematic review and meta-analysis have some limitations, which we address below. First, all included observational studies of our meta-analysis relied on the diagnosis criteria of primary studies included to identify dementia but there was substantial heterogeneity across the studies in the main analysis of dementia. Although this could be partly explained by different study design, dose, and duration of benzodiazepine use, we could not thoroughly explore other possible sources of variability due to the small number of included studies. Therefore, the pooled effect and heterogeneity assessment may be imprecise, and the publication bias of these studies could not be adequately addressed [35]. Second, our overall estimates displayed significant heterogeneity, so that any conclusion drawn has to be taken with caution. Heterogeneity was found for gender, geographical location, BMI, smoking status, comorbidity and co-medication, and dementia assessment methods. Although we used the appropriate meta-analysis technique with random-effects model, it cannot account for these differences. Also, we were not able to test for methodological variation. Third, our results were based on the analysis of observational studies. Therefore, it is impossible to exclude the possibility of potential confounding by various factors, which also associated with dementia among the elderly population. Finally, the possibility of publication bias is inherent in any meta-analysis of published data, because of the fact that often studies with null results tend not to be published [36]. Publication bias may have resulted in an overestimation of the relationship between benzodiazepine use and the risk of dementia.

\section{Public Health Implication}

Vascular dementia accounts for $24-48 \%$ of dementing illness in the older patients, an incidence of 6-10 in 10,000 per year in those older than 70 years [1]. The prevalence of dementia increased exponentially with age from $12.7 \%$ per year in the $90-94$ years of age, to $21.2 \%$ per year in the 
95-99 years of age, to $40.7 \%$ per year in the $100+$ years of age [37]. Age is the proverbial effect modifier of increasing dementia prevalence but age was not stratified by subgroup in the current review. Some studies found an association between parental age at birth and the risk of dementia, and mentioned that this might occur because of chromosomal abnormities [38, 39]. Similarly, BMI and obesity are also associated with diabetics and dementia [40]. Fitzpatrick et al. [41] demonstrated an increased risk of dementia among the obese $(\mathrm{BMI}>30)$ as compared to people with normal weight (BMI 20-25) at 50 years of age. They also reported a reverse association between BMI and the risk of dementia at $>65$ years of age [41]. This metaanalysis also found an inverse association between BMI of $>30$ and the risk of dementia among the elderly population. Several studies also found a positive association between smoking status and dementia risk [42, 43], which was also supported by our sensitivity analysis. Therefore, smoking could be a potential confounder for the association of cerebrovascular disease with dementia. In the case of drinking status, O'Keefe et al. [44] mentioned that alcohol consumption seemed to protect older people from dementia. To support this view, Luchsinger et al. [45] found an inverse association between wine consumption and dementia among individual age $>65$ years without the APOEe4 allele. In our meta-analysis, we found a positive relationship between alcohol consumption and the risk of dementia, which is also supported by some research [46].

\section{Recommendation}

There is currently no treatment for dementia although some drugs and non-drug treatments can improve both cognitive and behavioral symptoms. While factors such as age and genetics cannot be changed, other factors such as physical activity, diet, social engagement, and the cessation of tobacco and alcohol consumption may improve the situation. Not only younger but also elder people should exercise as much as they can because it may directly benefit the brain by increasing blood and oxygen flow into the brain. Accordingly, a report demonstrated an inverse association between physical activities and cognitive decline [47] and also mentioned that an increase level of physical activity is associated with a subsequently decreased incidence of dementia [48]. To reduce the chance of dementia, people should quit smoking because when compared with non-smokers, smokers were found to have decreased grey matter density in the posterior cingulate and presumes, right thalamus, and frontal cortex [49]. Foods such as red meats, grains, fruits, vegetables, fish, shellfish, and nuts which have an impact on brain health through their effect on cardiovascular health may also prevent dementia. Social engagement could improve cognitive functioning although Fratiglioni et al. [50] reported mixed results for the association between social networks and cognitive decline. To consider the increasing evidence of the potential adverse effects of benzodiazepines, regulatory agencies should provide guidelines for prescription, and physicians and other care givers should perform cognitive tests before prescribing this drug class for the general population.

\section{Conclusion}

The present study was designed to determine the effect of benzodiazepine use in the elderly population. On the basis of our systematic review and meta-analysis, we suggest that long-term use of benzodiazepines is associated with a higher dementia risk albeit with significant heterogeneity. To confirm or refute the results of our meta-analysis, a larger long-term prospective randomized controlled trial specifically designed to assess the effect of benzodiazepine use on the risk of developing dementia is needed.

\section{Disclosure Statement}

None.

\section{Acknowledgment}

This research is sponsored in part by Ministry of Science and Technology (MOST) under grant MOST 103-2221-E-038-014-, MOST 103-2221-E-038-016-, MOST 104-2221-E-038-013, MOST 104-3011-E-038-001, Health and Welfare Surcharge of Tobacco Products grant MOHW104-TDU-B-212-124-001, Ministry of Education, Taiwan, under grant TMUTOP103006-6.

\section{References}

Islam/Iqbal/Walther/Atique/Dubey/

Nguyen/Poly/Masud/Li/Shabbir
1 McCullagh CD, Craig D, McIlroy SP, Passmore AP: Risk factors for dementia. Adv Psychiatr Treat 2001;7:24-31.

2 Catindig JA, Venketasubramanian N, Ikram MK, Chen C: Epidemiology of dementia in Asia: insights on prevalence, trends and novel risk factors. J Neurol Sci 2012;321:11-16.

3 World Health Organization: Dementia: A Public Health Priority. Geneva, World Health Organization, 2012.

4 Ferri $\mathrm{CP}$, Prince M, Brayne C, et al: Global prevalence of dementia: a Delphi consensus study. Lancet 2006;366:2112-2117. 
5 Plassman BL, Langa KM, Fisher GG, et al: Prevalence of dementia in the United States: the aging, demographics, and memory study. Neuroepidemiology 2007;29:125-132.

6 Shively S, Scher AI, Perl DP, Diaz-Arrastia R: Dementia resulting from traumatic brain injury: what is the pathology? Arch Neurol 2012;69:1245-1251.

7 Saczynski JS, Beiser A, Seshadri S, et al: Depressive symptoms and risk of dementia: the Framingham Heart Study. Neurology 2010;75:35-41.

8 Roman GC: Stroke, cognitive decline and vascular dementia: the silent epidemic of the 21st century. Neuroepidemiology 2003;22:161164.

9 Kennelly SP, Lawlor BA, Kenny RA: Blood pressure and dementia - a comprehensive review. Ther Adv Neurol Disord 2009;2:241-260.

10 Linden M, Bär T, Helmchen H: Prevalence and appropriateness of psychotropic drug use in old age: results from the Berlin Aging Study (BASE). Int Psychogeriatr 2004;16:461-480.

11 Rickels K: Should benzodiazepines be replaced by antidepressants in the treatment of anxiety disorders? Fact or fiction? Psychother Psychosom 2013;82:351-352.

12 Stahl SM: Don't ask, don't tell, but benzodiazepines are still the leading treatments for anxiety disorder. J Clin Psychiatry 2002;63:756757.

13 Mamdani M, Rapoport M, Shulman KI, et al: Mental health-related drug utilization among older adults: prevalence, trends, and costs. Am J Geriatr Psychiatry 2005;13:892-900.

14 Hogan DB, Maxwell CJ, Fung TS, Ebly EM: Prevalence and potential consequences of benzodiazepine use in senior citizens: results from the Canadian Study of Health and Aging. Can J Clin Pharmacol 2003;10:72-77.

15 Liberati A, Altman DG, Tetzlaff J, et al: The PRISMA statement for reporting systematic reviews and meta-analyses of studies that evaluate health care interventions: explanation and elaboration. Ann Intern Med 2009; 151:W65-W94.

16 Higgins JP, Thompson SG, Deeks JJ, Altman DG: Measuring inconsistency in meta-analyses. BMJ 2003;327:557-560.

17 Shih HI, Lin CC, Tu YF, et al: An increased risk of reversible dementia may occur after zolpidem derivative use in the elderly population: a population-based case-control study. Medicine (Baltimore) 2015;94:e809.

18 Imfeld P, Bodmer M, Jick SS, Meier CR: Benzodiazepine use and risk of developing $\mathrm{Al}$ zheimer's disease or vascular dementia: a casecontrol analysis. Drug Saf 2015;38:909-919.

19 Lagnaoui R, Bégaud B, Moore N, et al: Benzodiazepine use and risk of dementia: a nested case-control study. J Clin Epidemiol 2002;55: 314-318.
20 Billioti de Gage S, Moride Y, Ducruet T, et al: Benzodiazepine use and risk of Alzheimer's disease: case-control study. BMJ 2014;349: g5205.

21 Wu CS, Wang SC, Chang IS, Lin KM: The association between dementia and long-term use of benzodiazepine in the elderly: nested case-control study using claims data. Am J Geriatr Psychiatry 2009;17:614-620.

$22 \mathrm{Wu}$ CS, Ting TT, Wang SC, et al: Effect of benzodiazepine discontinuation on dementia risk. Am J Geriatr Psychiatry 2011;19:151159.

23 Gray SL, Dublin S, Yu O, et al: Benzodiazepine use and risk of incident dementia or cognitive decline: prospective population based study. BMJ 2016;352:i90.

24 Gallacher J, Elwood P, Pickering J, et al: Benzodiazepine use and risk of dementia: evidence from the Caerphilly Prospective Study (CaPS). J Epidemiol Community Health 2012;66:869-873.

25 Billioti de Gage S, Bégaud B, Bazin F, et al: Benzodiazepine use and risk of dementia: prospective population based study. BMJ 2012;345:e6231.

26 Shash D, Kurth T, Bertrand M, et al: Benzodiazepine, psychotropic medication, and dementia: a population-based cohort study. Alzheimers Dement 2016;12:604-613.

27 Higgins JP, Green S (eds): Cochrane Handbook for Systematic Reviews of Interventions Version 5.1.0. The Cochrane Collaboration, 2011.

28 Zhong G, Wang Y, Zhang Y, Zhao Y: Association between benzodiazepine use and dementia: a meta-analysis. PLoS One 2015; 10:e0127836.

29 Lara VP, Caramelli P, Teixeira AL, et al: High cortisol levels are associated with cognitive impairment no-dementia (CIND) and dementia. Clin Chim Acta 2013;423:18-22.

30 Justin BN, Turek M, Hakim AM: Heart disease as a risk factor for dementia. Clin Epidemiol 2013;5:135-145.

31 Yamada M, Kasagi F, Sasaki H, et al: Association between dementia and midlife risk factors: the radiation effects research foundation adult health study. J Am Geriatr Soc 2003;51: 410-414.

32 Posner HB, Tang MX, Luchsinger J, et al: The relationship of hypertension in the elderly to $\mathrm{AD}$, vascular dementia, and cognitive function. Neurology 2002;58:1175-1181.

33 Craft S: Insulin resistance and Alzheimer's disease pathogenesis: potential mechanisms and implications for treatment. Curr Alzheimer Res 2007;4:147-152.

34 Johansson L, Guo X, Waern M, et al: Midlife psychological stress and risk of dementia: a 35-year longitudinal population study. Brain 2010;133(pt 8):2217-2224.
35 Higgins JP, Green S: Cochrane Handbook for Systematic Reviews of Interventions. Wiley Online Library, 2008.

$36 \mathrm{Lu} \mathrm{L}, \mathrm{Fu} \mathrm{Dl}$, Li HQ, et al: Diabetes and risk of Parkinson's disease: an updated meta-analysis of case-control studies. PLoS One 2014; 9:e85781.

37 Corrada MM, Brookmeyer R, Paganini-Hill $A$, et al: Dementia incidence continues to increase with age in the oldest old: the 90+ study. Ann Neurol 2010;67:114-121.

38 Borenstein AR, Copenhaver CI, Mortimer JA: Early-life risk factors for Alzheimer disease. Alzheimer Dis Assoc Disord 2006;20:63-72.

39 Ptok U, Papassotiropoulos A, Maier W, Heun $\mathrm{R}$ : Advanced parental age: a risk factor for $\mathrm{Al}$ zheimer's disease or depression in the elderly? Int Psychogeriatr 2000;12:445-451.

40 Luchsinger JA, Patel B, Tang MX, et al: Measures of adiposity and dementia risk in elderly persons. Arch Neurol 2007;64:392-398.

41 Fitzpatrick AL, Kuller LH, Lopez OL, et al: Midlife and late-life obesity and the risk of dementia: cardiovascular health study. Arch Neurol 2009;66:336-342.

42 Peters R, Poulter R, Warner J, et al: Smoking, dementia and cognitive decline in the elderly, a systematic review. BMC Geriatr 2008;8:36.

43 Aggarwal NT, Bienias JL, Bennett DA, et al: The relation of cigarette smoking to incident Alzheimer's disease in a biracial urban community population. Neuroepidemiology 2006;26:140-146.

44 O'Keefe JH, Bybee KA, Lavie CJ: Alcohol and cardiovascular health: the razor-sharp double-edged sword. J Am Coll Cardiol 2007;50: 1009-1014.

45 Luchsinger JA, Tang MX, Siddiqui M, et al: Alcohol intake and risk of dementia. J Am Geriatr Soc 2004;52:540-546.

$46 \mathrm{Xu} \mathrm{G}$, Liu X, Yin Q, et al: Alcohol consumption and transition of mild cognitive impairment to dementia. Psychiatry Clin Neurosci 2009;63:43-49.

47 Abbott RD, White LR, Ross GW, et al: Walking and dementia in physically capable elderly men. JAMA 2004;292:1447-1453.

48 Mehlig K, Skoog I, Waern M, et al: Physical activity, weight status, diabetes and dementia: a 34-year follow-up of the population study of women in Gothenburg. Neuroepidemiology 2014;42:252-259.

49 Almeida OP, Garrido GJ, Lautenschlager NT, et al: Smoking is associated with reduced cortical regional gray matter density in brain regions associated with incipient Alzheimer disease. Am J Geriatr Psychiatry 2008;16:9298.

50 Fratiglioni L, Paillard-Borg S, Winblad B: An active and socially integrated lifestyle in late life might protect against dementia. Lancet Neurol 2004;3:343-353. 